\title{
Effect of the Maillard Reaction on Functional Properties of Chitosan
}

\author{
Munehiko Tanaka, ${ }^{* 1}$ Jin Ru Huang, ${ }^{* 1}$ Wen Kuei Chiu, ${ }^{* 2}$ \\ Shoichiro Ishizaki, ${ }^{* 1}$ and Takeshi Taguchi ${ }^{* 1}$ \\ (Received May 21, 1993)
}

\begin{abstract}
In order to elucidate the possible participation of chitosan in the Maillard reaction, model systems consisting of chitosan and glucose $(1: 0.1,1: 0.5$, and $1: 1$, w/w) with different water activity (Aw 0.33, 0.51, and 0.84) were prepared. By heating the model systems at $65^{\circ} \mathrm{C}$, it was discovered that chitosan took part in the Maillard browning reaction and its rate was faster with a higher ratio of glucose and water activity. Furthermore, it was disclosed that some of the unique functional properties of chitosan were altered as a result of the Maillard reaction with glucose. Water binding capacity and antibacterial activity of chitosan decreased to some extent at the initial stage of the Maillard reaction, while acidic dye binding capacity increased considerably with the progress of the reaction. Most of the changes due to the Maillard reaction became significant when more than $80 \%$ of amino groups of chitosan were lost by the reaction. On the contrary, the fat binding capacity and iron chelating properties were not affected by the reaction.
\end{abstract}

It is well known that chitin, poly- $\beta(1 \rightarrow 4)-\mathrm{N}$ acetyl-D-glucosamine, one of the most abundant organic compounds on earth, is found predominantly in marine crustaceans, insects, and fungi. According to a review by Knorr, ${ }^{1)}$ the amount of chitin discarded as a waste product from the seafood processing industry is estimated to be approximately $1.2 \times 10^{5}$ metric tons annually on a worldwide basis. However, despite the quantitative importance of chitin, its industrial application has been limited.

On the other hand, chitosan, which is a linear polyamine, can be produced by the alkaline deacetylation of chitin. The unique physical, chemical and biological properties of the positively charged chitosan in acidic solution give rise to an enormous poten ial in industrial, cosmetic, medical, and food-related areas. For instance, in agriculture chitosan can act as a carrier for the controlled release of fertilizers and/or pesticides ${ }^{2)}$ and as a cationic flocculant in waste water treatment in the food industry. ${ }^{3,4}$ In pharmaceutical areas, chitosan is employed as an antimicrobial agent and also as encapsulation substance for drug ingredients..$^{5,83}$

Since most of the commercially available high molecular weight hydrocolloids or polysaccharides which are currently used in industries are neutral or polyanionic, chitosan, the cationic polyelectrolyte, is one of few exceptions. As a consequence, chitosan provides a great variety of potential applications. In view of the utilization of chitosan in food-related areas, an abundance of works have been carried out on functional properties of chitosan such as water uptake, ${ }^{7}$ lipid absorbing capacity, ${ }^{7)}$ emulsifying property, ${ }^{7}$ ) heavy metal uptake, ${ }^{8)}$ dye binding ability, ${ }^{8)}$ film forming property, ${ }^{10)}$ and antimicrobial activity. ${ }^{11}$

On the other hand, the Maillard reaction is one of the most prevalent chemical reactions occurring during food processing and preservation. As a result of the Maillard reaction, the development of brown color, the formation of desirable or undesirable flavor, a change of texture, and a loss of nutritional value are usually brought about. ${ }^{12,13}$ ) As pointed out above, chitosan has a large number of free amino groups in its molecule, which signifies the very possible participation of chitosan in the Maillard reaction. However, there has been no previous research published on the Maillard browning reaction of chitosan of which the authors are aware.

In this paper, model systems consisting of chitosan and glucose were prepared and the effect

*1 Department of Food Science and Technology, Tokyo University of Fisheries, Konan, Minato, Tokyo 108, Japan (田中宗彦, 黄 俊需, 石崎松一郎, 由口 武: 東京水産大学食品生痤学科).

*2 National Pingtung Polytechnic Institute, Pingtung Hsien, Taiwan（卭 文費：国立姩束技術学院）。 
of the Maillard reaction on functional properties of chitosan was studied.

\section{Materials and Methods}

\section{Preparation of the Model Systems}

Chitosan used in this study (degree of deacetylation =approximately $80 \%$ ) was obtained from Tokyo Kasei Kogyo, Ltd. and was ground in a Wiley Lab Mill to pass through a 40 mesh screen. The model systems were prepared by dispersing chitosan powder in a glucose solution and the mixtures were freeze-dried. The ratios of chitosan and glucose were adjusted to be $1: 0.1,1: 0.5$, and $1: 1$ by weight. The freeze-dried sample was crushed with a mortar and pestle to a fine powder and then equilibrated to the desired water activity $A(w)$ in vacuo over an appropriate saturated salt solution. For the adjustment of the water activity, saturated solutions of $\mathrm{MgCl}_{2}$ (Aw 0.33), $\left[\mathrm{Mg}\left(\mathrm{NO}_{3}\right)_{2}\right](\mathrm{Aw} 0.51)$, and $\mathrm{KCl}(\mathrm{Aw} 0.84)$ were used. The samples were allowed to equilibrate for 7 days at room temperature until the proper water activity was obtained.

Samples equilibrated to given water activities were heated at $65^{\circ} \mathrm{C}$ for up to $48 \mathrm{~h}$. For heating purposes, $10 \mathrm{~g}$ of the sample was placed in a glass vial $(3.0 \mathrm{~cm}$ i.d. $\times 8.0 \mathrm{~cm})$ and sealed with a screw cap. At given time intervals, samples were withdrawn and cooled in an ice-water bath.

\section{Analytical Procedures}

Determination of Brown Color: tristimulus color values of the model systems were determined by a Digital Color and Color Difference Meter (Model 101D, Nippon Denshoku Kogyo Co.) standardized with a white color plate $(L=90.9, a=0, b=3.1)$.

Free Amino Groups in Chitosan: the amount of free amino groups in chitosan was determined by the colloidal titration method using N/400 potassium polyvinylsulfate (Wako Pure Chemical Ind.) with $0.1 \%$ tolouidine blue (Wako Pure Chemical Ind.) as an indicator.

Glucose: the amount of glucose in the model systems was measured by the enzymatic method employing Glucose B-Test (Wako Pure Chemical Ind.).

Water Uptake: the water binding capacity of chitosan was determined according to the procedure of Sosulski ${ }^{14}$ with a $5 \mathrm{~g}$ sample and $30 \mathrm{ml}$ of distilled water.

Fat Binding Property: fat binding capacity was determined by the method of Lin et $a l .^{15}$ using
$0.5 \mathrm{~g}$ chitosan and $3 \mathrm{~m} /$ corn oil in a $15-\mathrm{m} /$ conical graduated centrifuge tube.

Dye Binding Property: allura Red (equivalent to FD \& C Red No. 40, Tokyo Kasei Kogyo, Ltd.) was used to determine the dye binding capacity of chitosan by the procedure of Knorr. ${ }^{\theta>}$

Absorption of Iron: $20 \mathrm{ml}$ of $\mathrm{FeCl}_{3}$ standard solution $(20 \mu \mathrm{gFe} / \mathrm{ml}$, Wako Pure Chemical Ind.) was mixed with $100 \mathrm{mg}$ of chitosan and stirred for $30 \mathrm{~min}$ at room temperature. After centrifugation at $500 \times \mathrm{g}$ for $10 \mathrm{~min}$, the amount of free ferric ion in the supernatant was determined by the $o$-phenanthroline method.

\section{Antibacterial Activity of Chitosan}

Strains: the following bacterial strains were used in this study: Alteromonas putrefaciens IAM 12089, Pseudomonas fuorescens IAM 12022, Escherichia coli IAM 12119, Vibrio parahaemolyticus S7, Staphylococcus aureus IAM 1098 and 209P, Bacillus cereus S6, B. megaterium IAM 1702, B. subtilis IAM 1026 and IAM 12118, Streptococcus lactis IAM 1198, Lactobacillus plantarm IAM 1216, Leuconostoc mesenteroides IAM 1046, and Pediococcus acidilactici IAM 10061. All bacterial strains were kindly provided from the Laboratory of Food Microbiology, Tokyo University of Fisheries.

Media: the medium for bacteria, except for lactic acid bacteria, consisted of $0.25 \%$ yeast extract, $0.5 \%$ polypeptone, $0.1 \%$ glucose, and $1.5 \%$ agar, pH 6.0 (Code 05618, Nissui Pharmaceutical Co. Ltd.). Three percent of $\mathrm{NaCl}$ was added to the above medium for the Vibrio strain. For lactic acid bacteria, the medium was composed of $0.55 \%$ yeast extract, $1.25 \%$ polypeptone, $1.1 \%$ glucose, $0.025 \% \mathrm{KH}_{2} \mathrm{PO}_{4}, 0.025 \% \mathrm{~K}_{2} \mathrm{HPO}_{4}$, $1 \% \mathrm{CH}_{3} \mathrm{COONa}, 0.01 \% \mathrm{MgSO}_{4}, 0.0005 \% \mathrm{MnSO}_{4}$, $0.0005 \% \mathrm{FeSO}_{4}$, and $2 \%$ agar, pH 6.0 (Code 05800, Nissui Pharmaceutical Co. Ltd.). Liquid versions of the above media were employed as the broth culture, and the dilution of inocular was made with sterile $0.85 \% \mathrm{NaCl}$ solution. Determination of Antibacterial Activity: chitosan was dissolved in a $0.5 \%$ acetic acid solution. After the adjustment of $\mathrm{pH}$ to 6.0 with $\mathrm{KOH}$, the chitosan solution was sterilized with Acrodisc syringe filter units $(0.4 \mu \mathrm{m}$, Gelman Sciences) and used immediately after preparation.

The agar dilution method was used throughout this study for the determination of antibacterial activity. Diluted $20 \mathrm{~h}$ broth culture of bacterium with approximately $10^{7}$ cells per $\mathrm{m} l$ was inoculated 
to the agar medium containing an appropriate concentration of chitosan or browned chitosan in the petri dishes. The plates were incubated at $37^{\circ} \mathrm{C}$ for $20 \mathrm{~h}$, except Pseudomonas fluorescens and lactic acid bacteria which were incubated at $30^{\circ} \mathrm{C}$. The extent of growth inhibition was followed by the naked eye.

\section{Results and Discussion}

\section{Development of Brown Color}

Figure 1 presents the development of brown color during heating of the mixture of chitosan and glucose at $65^{\circ} \mathrm{C}$. Tristimulus color value $L$ was used as an index of the color development, because the change in $L$ value was more apparent than that of $a$ or $b$ value. It is evident from this Figure that chitosan takes part in the Maillard reaction in the presence of glucose, since chitosan per se did not produce any brown color in the absence of glucose. Though an induction period of approximately $4 \mathrm{~h}$ was observed in the model
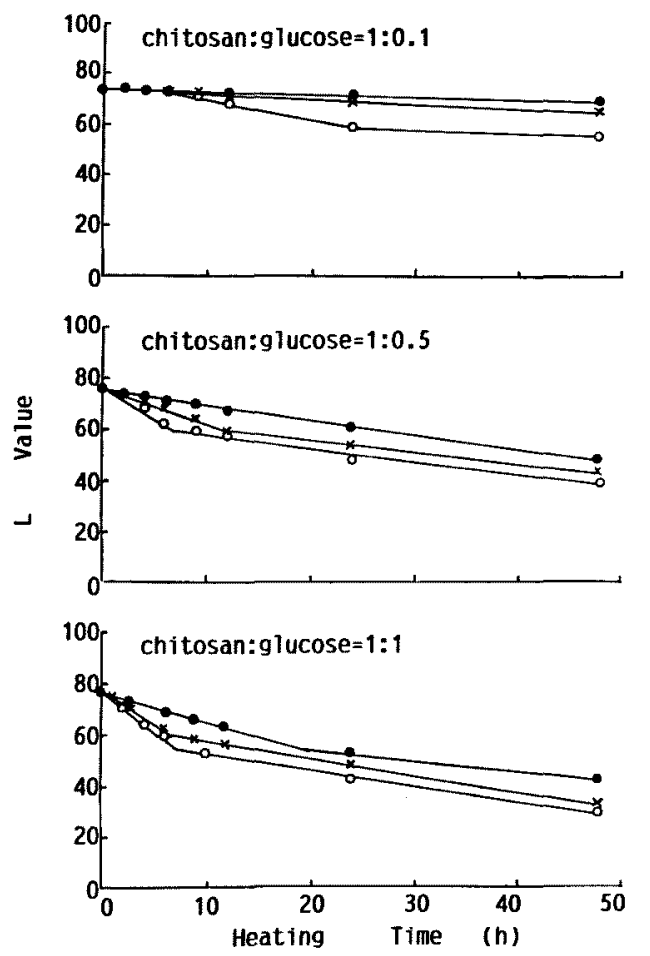

Fig. 1. Development of brown color during the Maillard reaction between chitosan and glucose at $65^{\circ} \mathrm{C}$.

-: Aw 0.33, x: Aw 0.51, O: Aw 0.84 system consisting of chitosan and glucose with a ratio of $1: 0.1$ (by weight), the model systems with 1: 0.5 and $1: 1$ did not have any induction period. The development of brown color followed the zero order reaction and its rate was faster with

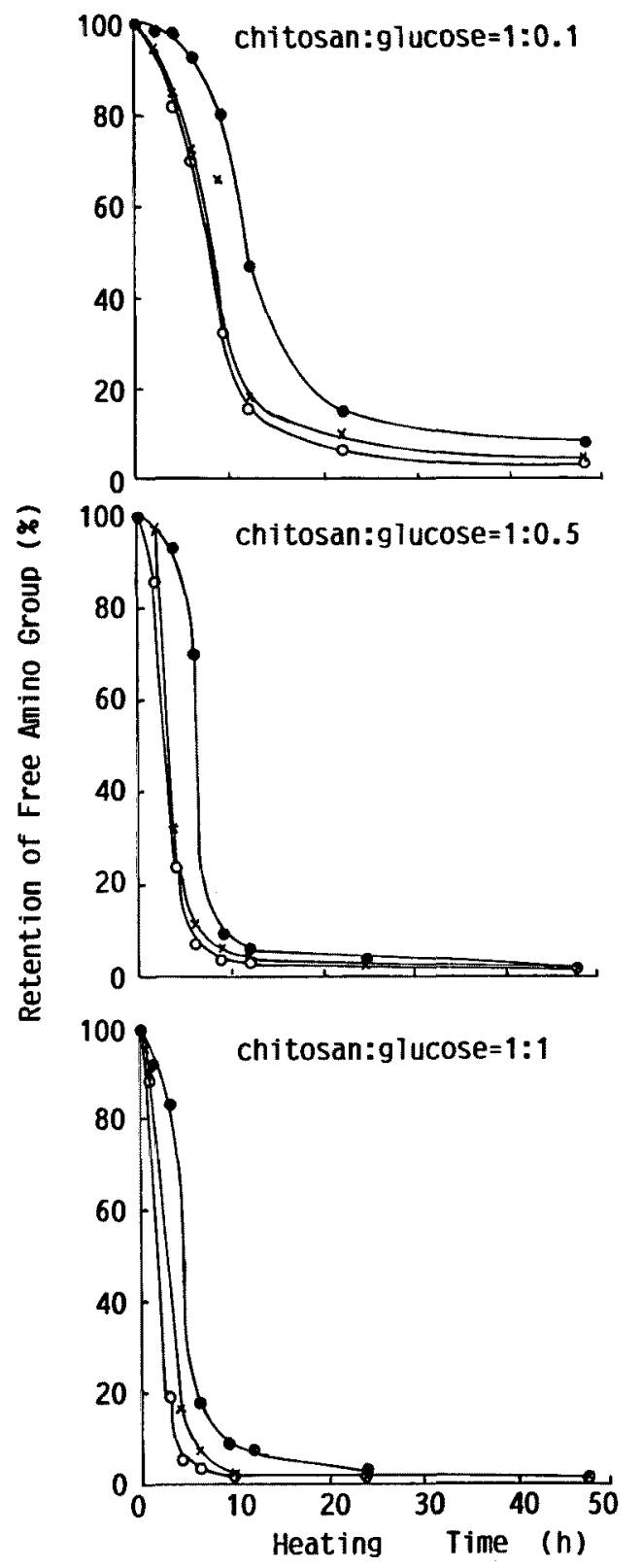

Fig. 2. Retention of free amino group of chitosan during the Maillard reaction with glucose at $65^{\circ} \mathrm{C}$.

๑: Aw 0.33, x: Aw 0.51, o: Aw 0.84 


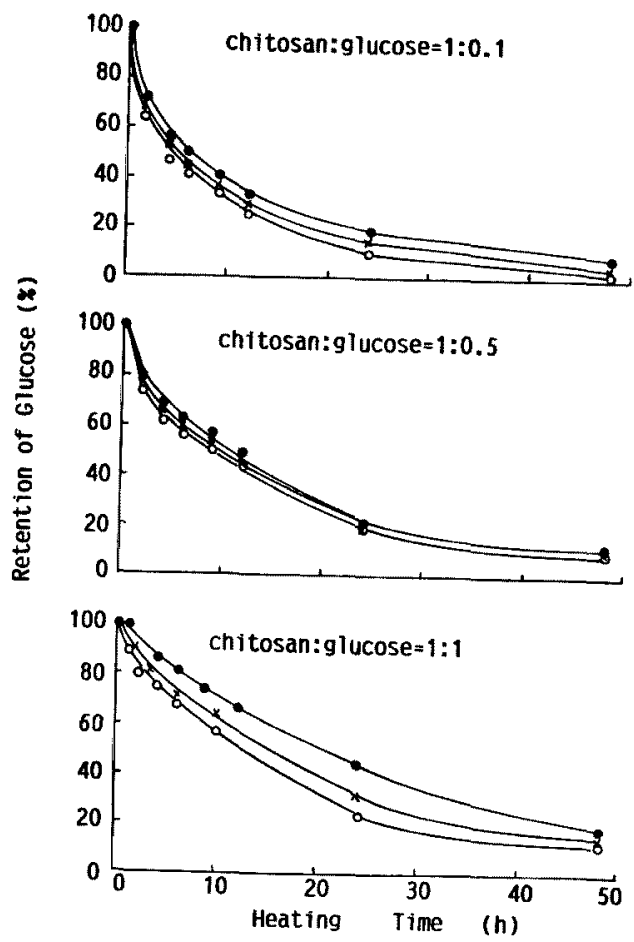

Fig. 3. Retention of glucose during the Maillard reaction with chitosan at $65^{\circ} \mathrm{C}$.

-: Aw $0.33, x: 0.51,0$ : Aw 0.84

an increasing ratio of glucose and water activity (Fig. 1).

\section{Loss of Free Amino Groups in Chitosan}

When the Millard reaction has taken place between chitosan and glucose, the amount of free amino groups in chitosan is expected to decrease. Therefore, in this study, the degree of deacetylation was determined by the colloidal titration method. Figure 2 depicts the retention of free amino groups of chitosan during its Maillard reaction at $65^{\circ} \mathrm{C}$. The amount of free amino groups in chitosan decreased rapidly at a very early stage of the reaction. However, the decreasing rate of free amino groups was faster with a higher glucose ratio and water activity. More than $80 \%$ of the amino groups were lost during the first $20 \mathrm{~h}$ in the model system at $1: 0.1$, and during $10 \mathrm{~h}$ at $1: 0.5$ and $1: 1$. It is noteworthy that the time giving the apparent development of brown color (Fig. 1) corresponded to the time most of free amino groups disappeared, suggesting that the combination of amino group with al- dehyde group of glucose preceded the development of brown color.

\section{Loss of Glucose}

The retention of free glucose as a function of the reaction time is shown in Fig. 3. It is obvious that the overall tendency of glucose loss was analogous to that of free amino group loss (Fig. 2). However the loss rate of glucose during the Maillard reaction decreased with the increasing ratio of glucose to chitosan in the model system and was considerably slower than that of the free amino group loss.

\section{Water Binding Capacity}

It is well known that chitosan is soluble in dilute organic acid but insoluble in neutral and alkaline aqueous solvents. Since most of the commercially available high molecular weight

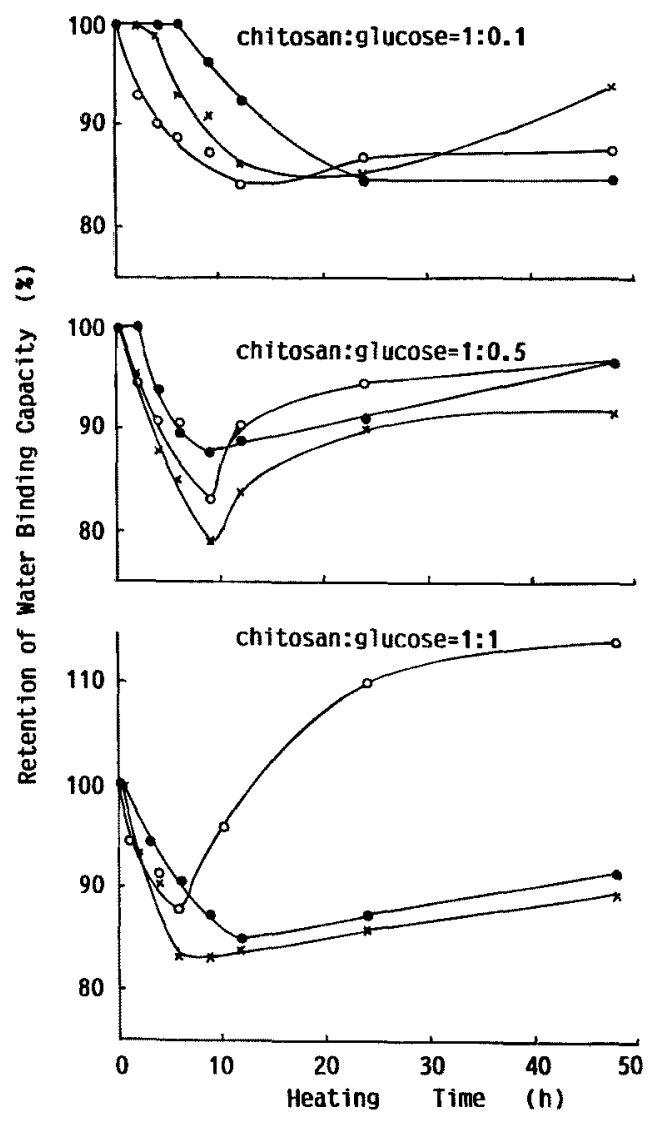

Fig. 4. Change in the water binding capacity of chitosan during the Maillard reaction with glucose at $65^{\circ} \mathrm{C}$.

: Aw 0.33, X: Aw 0.51, O: Aw 0.84 
hydrocolloids are neutral or polyanionic, chitosan has a wide range of application in food industries due to its cationic nature. Knorr ${ }^{*)}$ determined the water binding capacities of chitosan and its structurally related substances such as chitin and microcrystalline cellulose, and discovered that the water uptake of chitin and chitosan was significantly larger than that of microcrystalline cellulose.

Figure 4 presents the changes in water binding capacity of chitosan during its Maillard reaction with glucose at $65^{\circ} \mathrm{C}$. The water binding capacity of chitosan before the reaction was found to be $412 \%(w / w)$, which is in agreement with the values reported by Knorr. ${ }^{7)}$ As is apparent from this Figure, the water binding capactity of chitosan decreased $15-20 \%$ in the initial stage of the reaction and reached the minimum points which coincided with the time required to about $90 \%$ loss of amino groups (Fig. 2). There appeared to be a marginal increase in water binding capacity in the later stage of the reaction, but it was never restored to its original capacity except for the model system at a 1:1 ratio with Aw 0.84. Form these results it was concluded that the water binding capacity of chitosan was slightly affected by the Maillard reaction.

\section{Fat Binding Capacity}

The fat binding capacity of chitosan used in this study was $200 \mathrm{~m} l$ per $100 \mathrm{~g}$, which is comparable to the result reported by Knorr. ${ }^{2}$ Although the data are not shown, the amount of corn oil absorbed by chitosan was not altered during the Maillard reaction at $65^{\circ} \mathrm{C}$ irrespective of the composition of the model systems. Therefore, it is suggested that free amino groups of chitosan might not be attributable to fat binding capacity.

\section{Dye Binding Property}

Changes in the dye binding capacity of chitosan during the Maillard reaction with glucose at $65^{\circ} \mathrm{C}$ are presented in Fig. 5. The dye uptake of chitosan used in this experiment was $171 \mu \mathrm{g} / \mathrm{g}$. Since chitosan has positively charged amino groups in its molecule, acidic dyes such as FD \& $C$ Red No. 40, ${ }^{97}$ Acid Orange II, ${ }^{18)}$ and Brilliant Yellow ${ }^{18)}$ are easily absorbed by chitosan under neutral conditions. On the other hand, the absorption of basic dyes such as Crystal Violet ${ }^{18)}$ is completely inhibited due to electronstatic repulsion.

It is obvious from Fig. 5 that the dye binding capacity of chitosan increased concomitantly with the development of the Maillard reaction. The

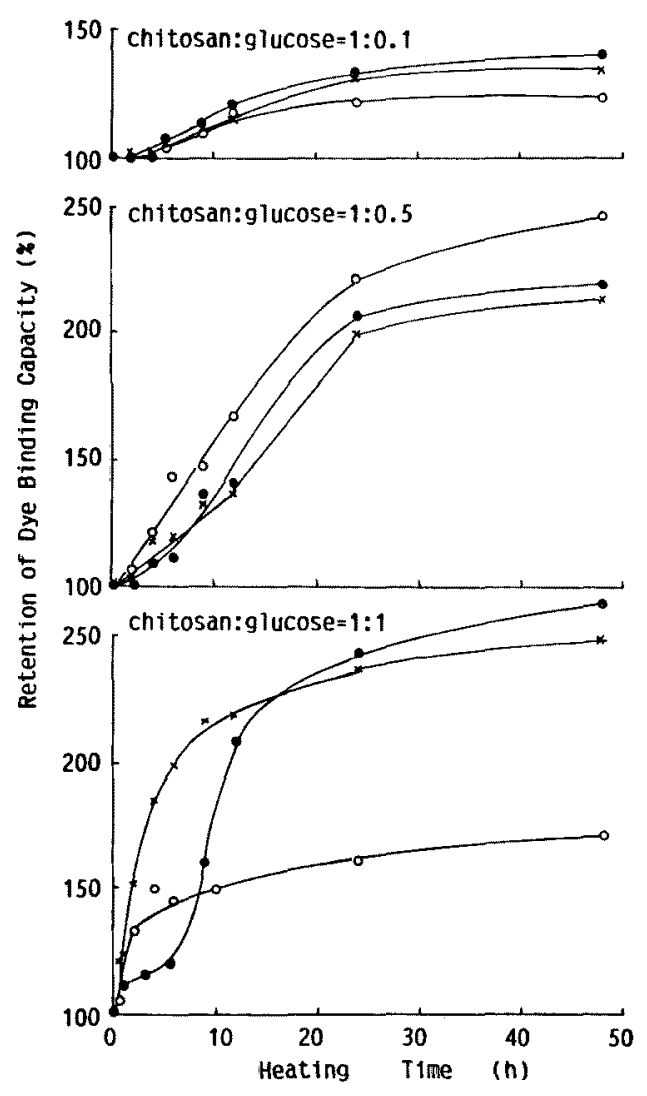

Fig. 5. Change in the dye binding capacity of chitosan during the Maillard reaction with glucose at $65^{\circ} \mathrm{C}$.

-: Aw $0.33, \times$, Aw $0.51,0$ : Aw 0.84

reason for the increase in capacity by the reaction is hard to understand at the moment, because the net positive charge of chitosan is expected to decrease as a result of the Millard reaction.

\section{Absorption of Iron}

The chelating ability of chitosan for metal ions, such as mercury, zinc, chromium, copper, lead, plutonium, and uranium has been well demonstrated.17-18) According to a report by Muzzarelli and Tubertin, $\left.{ }^{20}\right)$ the most strongly absorbed metal is copper, followed by nickel, zinc, cobalt, iron, and manganese. The amount of iron bound by chitosan in this study was $3.82 \mathrm{mg} / \mathrm{g}$, and this was not changed by the development of the Maillard reaction (data not shown). This suggests that a free amino group of chitosan may not be involved in the absorption mechanism of iron. 
Table 1. Sensitivity of bacterial strains to chitosan*1

\begin{tabular}{|c|c|c|}
\hline \multirow{2}{*}{ Strain } & \multicolumn{2}{|c|}{ Percentage of survivors } \\
\hline & Chitosan & Browned chitosan*2 \\
\hline Alteromonas putrefaciens (IAM 12089) & $-\ldots$ & - \\
\hline Pseudomonas fluorescens (IAM 12022) & - & - \\
\hline Escherichia coli (IAM 12119) & - & - \\
\hline Staphylococcus aureus (IAM 1098) & - & - \\
\hline Staphylococcus aureus (209P) & 10.8 & 44.1 \\
\hline Vibrio parahaemolyticus (S7) & 9.0 & 16.7 \\
\hline Bacillus cereus (S6) & 0 & 2.3 \\
\hline Bacillus megaterium (IAM 1702) & 18.0 & 30.3 \\
\hline Bacillus subtilis (IAM 1026) & 4.0 & 15.0 \\
\hline Bacillus subtilis (IAM 12118) & 11.9 & - \\
\hline Lactobacillus plantarm (IAM 1216) & - & - \\
\hline Leuconostoc mesenteroides (IAM 1046) & 0 & 1.1 \\
\hline Pediococcus acidilactici (IAM 10061) & - & - \\
\hline Streptococcus lactis (IAM 1198) & 5.2 & 40.2 \\
\hline
\end{tabular}

*1 Agar dilution method: $37^{\circ} \mathrm{C}$ for $20 \mathrm{~h}, \mathrm{pH} 6.0 ;$ Sample: $100 \mu \mathrm{g} / \mathrm{ml}$ medium.

*2 Browned chitosan was prepared by heating chitosan in the presence of glucose $\left(1: 0.5\right.$, w/w) at Aw $0.84,65^{\circ} \mathrm{C}$ for $6 \mathrm{~h}$.

*3 Indicates that the antibacterial activity was not detected under the conditions used in this study.

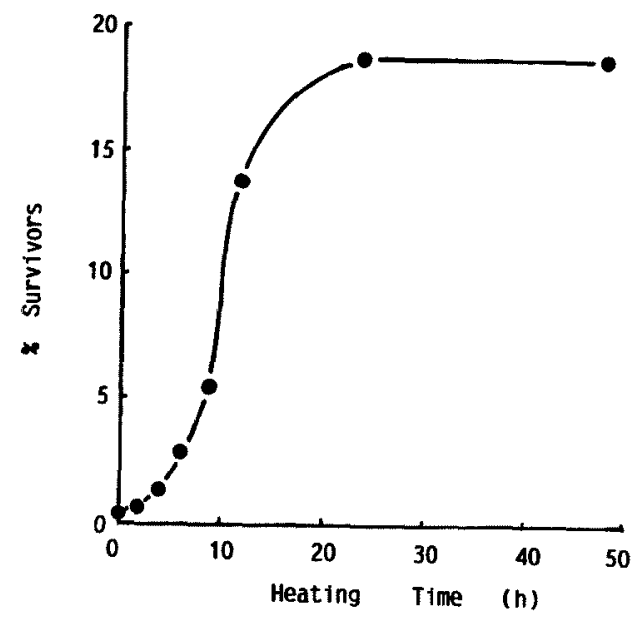

Fig. 6. Changes in the antibacterial activity of chitosan against $B$. subtilis (IAM 1026) during the Maillard reaction in the presence of glucose at $65^{\circ} \mathrm{C}$, Aw 0.51 .

Chitosan: Glucose $=1: 0.5(\mathrm{w} / \mathrm{w})$.

The amount of chitosan used for the assay was $100 \mu \mathrm{g} / \mathrm{ml}$ medium. Inocula used for the assay was $3.1 \times 10^{3}$.

\section{Antibacterial Activity}

In this study, the antibacterial activity of chitosan was determined as \% survivors of $\boldsymbol{B}$. subtilis (IAM 1026), that is, the smaller the percentage of survivors, the greater the antibacterial activity. The model system consisting of chitosan and glucose $(1: 0.5, \mathrm{w} / \mathrm{w})$ with Aw 0.51 was subjected to the Maillard reaction at $65^{\circ} \mathrm{C}$ for up to $48 \mathrm{~h}$. The amount of inocula used for the assay was $3.1 \times 10^{5}$ and the amount of chitosan used was $100 \mu \mathrm{g}$ per $\mathrm{m} l$ of medium.

Figure 6 illustrates the change in the antibacterial activity of chitosan against $B$. subtilis during the Maillard reaction. The antibacterial activity decreased at the incipient stage of the reaction and plateaued out at the later stage. It is noteworthy that the decrease in antibacterial activity became apparent when most of the free amino groups of chitosan disappeared (Fig. 2), indicating that a free amino group might play an important role in antibacterial activity. On the other hand, it is further suggested that the antibacterial function of chitosan is not solely dependent on its free amino groups, since browned chitosan with only a slight amount of amino group (Fig. 2) still possessed some antibacterial activity (Fig. 6). More detailed studies are needed to reveal the mechanism of antibacterial activity.

Table 1 lists the sensitivity of some bacterial strains to chitosan before and after the Maillard reaction. Chitosan did not have activity against Gram negative bacteria used in this study except Vibrio and its sensitivity to Gram positive bacteria varied. Furthermore, the growth of Staphylococcus aureus IAM 1098 was not inhibited by chitosan, while that of its different strain (209P) was suppressed to some extent. The sensitivity of $B$. subtilis also varied considerably from strain to strain. It was further revealed that the antibacterial ac- 
tivity of chitosan was more or less lost in the progress of the Maillard reaction.

In conclusion, this study points out that chitosan powder undergoes the Maillard reaction in the presence of glucose and that some of its functional properties are consequently altered. Brown color was developed following the zero order reaction and most of the free amono groups of chitosan disappeared at the initial stage of the reaction. It is interesting to note that the water binding capacity and antibacterial activity of chitosan were lost as a result of the reaction, whereas dye binding capacity increased significantly. However, fat binding capacity and iron chelating property were not altered by the reaction. In order to utilize chitosan as an ingredient in food processing based on its unique functions, it is necessary to consider the possible occurrence of the Maillard browning reaction.

\section{Acknowledgment}

The authors are grateful to Prof. T. Fujii, Tokyo University of Fisheries, for providing the bacterial strains used in this study.

\section{References}

1) D. Knorr: Recovery and utilization of chitin and chitosan in food processing waste management. Food Technol, 45, 114-122 (1991).

2) S. Hirano, H. Senda, Y. Yamamoto, and A. Watanabe: Several novel attempts for the use of the potential functions of chitin and chitosan. In "Chitin, Chitosan, and Related Enzymes", Academic Press, New York, 1984, pp. 77-95.

3) I. G. Dosio, R. A. Fisher, and P. A. Carroad: Bioconversion of shellfish chitin waste: Waste pretreatment, enzyme production, process design, and economic analysis. J. Food Sci., 47, 901-905 (1982).

4) T. C. Yang and R. R. Zall: Absorption of metals by natural polymers generated from seafood processing wastes. Ind.
Eng. Chem. Prod. Nes. Dev., 23, 168-172 (1984).

5) K. D. Vorlop and J. Klein: Formation of spherical chitosan biocatalysts by ionotropic gelation. Biotechnol. Lett., 3, 914 (1981).

6) A. El Ghaouth, J. Grenier, and A. Asselin: Antifungal activity of chitosan on two postharvest pathogens of strawberry fruits. Phytopathology, 82, 398-402 (1992).

7) D. Knorr: Functional properties of chitin and chitosan. J. Food Sci. 47, 593-595 (1982)

8) T. Mitani, A. Moriyama, and $H$, Ishii: Heavy metal uptake by swollen chitosan beads. Biosci. Biotech., Biochem., 56, 985 (1992).

9) D. Knorr: Dye binding properties of chitin and chitosan. J. Food Sci., 48, 36-37+41 (1983).

10) D. W. S. Wong, F. A. Gastieau, K. S. Gregorski, S. J. Tillin, and A. E. Pavlath: Chitosan-lipid films: Microstructure and surface energy. J. Agric. Food Chem, 40, 540-544 (1992).

11) G. G. Allan, L. C. Altman, R. E. Bensinger, D. K. Ghosh, Y. Hirabayashi, A. N. Neogi, and S. Neogi: Biomedical applications of chitin and chitosan. In "Chitin, Chitosan, and Related Enzymes", Academic Press, New York, 1984, pp. 119-133.

12) T. C. Lee, M. Kimiagar, S. J. Pintauro, and C. O. Chichester: Physiological and safety aspects of Maillard browning of foods. Prog. Food Nutr. Sci, 5, 243-256 (1981).

13) M. Namiki: Chemistry of Maillard reaction: Recent studies on the browning reaction mechanism and the development of antioxidants and mutagens. Adv. Food Nes., 32, 115-185 (1988).

14) F.W. Sosulski: The centrifuge method for determining flour absorption in hard red spring wheats. Cereal Chem., 39, 344-350 (1962).

15) M. J. Y. Lin, E. S. Humbert, and F. W. Sosulski: Certain functional properties of sunflower meal products. $J$. Food Sci., 39, 368-370 (1974).

16) H. Yoshida: Applications of chitin and chitosan. Kagaku to Kogyo, 64, 325-330 (1990).

17) C. A. Eiden, C. A. Jewell, and J. P. Wightman: Interaction to lead and chromium with chitin and chitosan. J. Appl. Polym. Sci, 25, 1587-1599 (1980).

18) D. Knorr: Use of chitinous polymers in food. Food Technol, 38, 85-97 (1984).

19) N. Nishi, Y. Maekita, S. Nishimura, O. Hasegawa, and $\mathbf{S}$. Tokura: Highly phosphorylated derivatives of chitin, partially deacetylated chitin and chitosan as new functional polymers: metal binding property of the insolubilized materials. Int. J, Biol. Macromol., 9, 109-114 (1987).

20) R. A. A. Muzzarelli and O. Tubertini: Purificaiton of thallium(I) nitrate by column chromatography. Microchim. Acta, 5, 892-899 (1970). 